\title{
Determination of Malondialdehyde and Superoxide Dismutase in Pulmonary Tuberculosis Subjects
}

\author{
Muhammad $Y^{1}$, Iliya $S^{2}$, Sa'idu $A Y^{3}$, Anka $A^{4}$, Umar $K^{4}$, Sani $Z^{5}$, Zainab $I^{6}$ \\ 1 Principal Medical Laboratory Scientist (PMLS), Department of Chemical Pathology, Rasheed Shekoni Teaching Hospital Dutse, Dutse, \\ Nigeria \\ 2 Department of Biological Sciences, School of Pure and Applied Sciences, Mount Kenya University Thika, Kenya \\ 3 Department of Biological Sciences, Faculty of Science, Federal University Dutse, Dutse, Jigawa State, Nigeria \\ 4 Department of Medical Laboratory Science, Ahmadu Bello University, Zaira, Nigeria \\ 5 Department of Science Laboratory Technology, Jigawa State Polytechnic, Dutse, Nigeria \\ 6 Department of Haematology and Blood Bank, Rasheed Shekoni Specialist Hospital, Dutse, Nigeria
}

*Corresponding Author: Dr. Muhammad Y, Principal Medical Laboratory Scientist (PMLS), Department of Chemical Pathology, Rasheed Shekoni Teaching Hospital Dutse, Dutse, Nigeria.

Email: yahyoukhan@gmail.com; Tel: +2348064731241

Received: January 14, 2020; Accepted: February 07, 2020

\begin{abstract}
Pulmonary tuberculosis (PTB) is a dangerous bacterial infection that attacks the lungs. It has long been documented that there is an increase circulating levels of free radicals and oxidative stress markers in TB subjects. Malnutrition and deficient antioxidant capabilities further complicate the patient's situation. The aim of this study is to determine the serum levels of malondialdehyde (MDA) and superoxide dismutase (SOD) in pulmonary tuberculosis patients and normal volunteers at Rasheed Shekoni Teaching Hospital Dutse, Jigawa State, Nigeria. The present study consists of one hundred and thirty-four (134) subjects, Ninety (90) among which are diagnosed TB patients and forty-four (44) apparently healthy controls attending Rasheed Shekoni Teaching Hospital Dutse. Venous samples were collected from ninety consecutive, consenting PTB on drugs and those that have not started medications. MDA levels were analysed using the method of Nadigar et al (1986). The mean values of MDA in the 24 tuberculosis infected males who are yet to commence drug was $4.0 \pm 0.32,2.8 \pm 0.53$ in those that are on drugs and $2.0 \pm 0.23$ in the male control subjects. It was $4.1 \pm 0.35,2.9 \pm 0.29$ and $1.9 \pm 0.32$ in female that are TB infected and yet to commence drugs, those on drugs and the control subjects respectively. The total mean MDA value for those on tuberculosis drugs was $4.0 \pm 0.33,2.8 \pm 0.46$ for those that are yet to commence the treatment and $2.0 \pm 0.28$ for the control subjects, the serum levels of SOD was found significantly lower $(p<0.005)$ in PTB subjects on treatment compared to those without treatment and control subjects. Conclusively, the findings of the current study showed that pulmonary tuberculosis patients are predisposed to oxidative stress leading to an increased MDA and consequent decreased SOD levels as compared to the control subjects.
\end{abstract}

Keywords: Pulmonary Tuberculosis, Antioxidant, Free radicals, Malondialdehyde, Superoxide Dismutase.

\section{INTRODUCTION}

Mycobacterium tuberculosis is a causative agent of pulmonary tuberculosis (PTB). It's an infectious disease ranked among major global health problem afflicting approximately 10 million people each year [1,2]. Tuberculosis incidence is predicted to be 615 per 100,000 in Nigeria ranking first in Africa and $22^{\text {nd }}$ high tuberculosis burden countries in the world [2]. PTB affects the lungs primarily but when the infections centred in several other parts of the body like (lymph nodes, bones, joints, skin, meninges, eyes, kidneys and gastro-intestinal tracts), it is considered as extrapulmonary tuberculosis [3]. PTB is characterised by persistent cough of more than two weeks, sputum production, anorexia, fatigue, haemoptysis, night sweat, dehydration, vomiting, shortness in breathing, loss of appetite, chest pains and fever [4,5]. TB infection is spread through inhalation of air from cough, spit or sneeze of infected individuals leading to asymptomatic or latent infection and later progressed to active infection [6].

Following infection of mycobacterium to the lungs, free radicals are produced either through respiratory burst mechanism of 
macrophages leading to the direct generation of enormous radicals or interfering with normal function of superoxide dismutase (SOD) which is highly expressed in the lungs $[7,8,9]$. SOD is one of the antioxidant proteins that catalyses the dismutation of superoxide anion to hydrogen peroxide $\left(\mathrm{H}_{2} \mathrm{O}_{2}\right)$ thereby limiting amount of hydroxyl radical, $\mathrm{H}_{2} \mathrm{O}_{2}$ is further converted to oxygen and water by catalase or glutathione peroxidase [10]. SOD participates in scavenging free radicals especially superoxide anion radical, therefore subsequent interference of its activities may lead to further propagation of radicals in the body [7]. In view of this, the body releases antioxidant substances serving as defensive mechanisms to counteract the effects of free radicals generated. Malnutrition reported with PTB patient may be additional problem complicating impaired antioxidant ability and immunity [11]. Oxidative stress may be defined as the situation whereby concentration of free radicals is higher than antioxidants [11,12]. These radicals usually attack delicate structures such as DNA forming adducts, polyunsaturated fatty acid (PUFA) in the cell membrane a process called lipid peroxidation [11, 12]. Malondialdehyde (MDA), a three-carbon molecular weight aldehyde is one of by-products of lipid peroxidation and is the most widely studied marker of oxidative stress [13].

The present study aimed at determining the level of MDA using TBARS (Thiobarbituric Acid Reactive Substances) in PTB patients (on drugs and those that are yet to start medications) and normal apparently healthy volunteers at Rasheed Shekoni Specialist Hospital Dutse and to advocate synergizing nutritional management in PTB patients.

\section{MATERIALS AND METHODS}

Study Population: The present study consists of one hundred and thirty-four (134) subjects, ninety (90) among which are diagnosed TB patients and forty-four (44) apparently healthy controls attending Rasheed Shekoni Teaching Hospital Dutse. The diagnosed TB patients are divided into 45 each, first categories are those on drugs and second group have not yet started medication.

Sampling Techniques: Ninety consecutive, consenting TB on drugs and those that have not started medications were recruited in this study.

Blood Specimen Collection: Venous samples were collected from the diagnosed TB patients and apparently healthy controls using standard venepuncture and delivered into well labelled clean test tubes. The blood samples were allowed to clot for 30 minutes and then centrifuged at 5,000 rpm for 5 minutes to obtain the serum; the separated sera were stored at $-20^{\circ} \mathrm{C}$.

Determination of MDA: MDA was determined in accordance of procedure described by Nadigar et al. (1986) [14]. This method was based on the principle that acetic acid detaches the lipid and protein of the tissue. Thiobarbituric acid reacts with lipid peroxide, hydroperoxide and oxygen labile double bond to form the colour adduct with absorption maxima at $530 \mathrm{~nm}$.

Determination of SOD: The activity of SOD was assayed in accordance with method of Kakkar et al., (1984) [15]. 2.5ml of $0.05 \mathrm{M}$ carbonate buffer was added into test tube labelled as test and blank respectively, $0.2 \mathrm{ml}$ of sample was added into the test while corresponding $0.2 \mathrm{ml}$ of distilled water was added into the blank tube and this was followed by the addition of $0.3 \mathrm{ml}$ of $0.03 \mathrm{mM}$ of adrenalin. They were then mixed and absorbance of test was read spectrophotometrically at $420 \mathrm{~nm}$ after zeroing the instrument with the blank

\section{Statistical Analysis:}

All the results were presented as Mean $\pm S D$. One-way analysis of variance (ANOVA) was conducted, followed by Turkey Kramer multiple comparison test using SPSS Software version 26.0 (why not version 26.0 since it is the latest version). Differences were considered as significant when $\mathrm{P}<0.05$.

\section{RESULTS}

The table 1.0 below shows the result of the findings, the mean value of MDA in the 24 Tuberculosis infected males who are yet to commence drug was $4.0 \pm 0.32,2.8 \pm 0.53$ in those that are drugs and $2.0 \pm 0.23$ in the male control subjects. It was $4.1 \pm 0.35$, $2.9 \pm 0.29$ and $1.9 \pm 0.32$ in female that are TB infected and yet to commence drugs, those on drugs and the control subjects respectively. The total mean MDA value for those on tuberculosis drugs was $4.0 \pm 0.33,2.8 \pm 0.46$ for those that are yet to commence the treatment and $2.0 \pm 0.28$ for the control subjects.

The table 2.0 shows the serum levels of SOD in PTB on ATT, those that have not commence medications and control subjects as $0.80 \pm 0.51,1.45 \pm 0.48$ and $2.05 \pm 0.85$ respectively.

Table 1: Serum levels of MDA in PTB subjects on drugs and those that have not started medications

\begin{tabular}{lll}
\hline PARAMETERS & GENDER & MDA(mmol/l) \\
\hline PTB SUBJECTS ON ATT & Male $\quad(n=23)$ & $4.0 \pm 0.32$ \\
& Female $(n=22)$ & $4.1 \pm 0.35$ \\
& Pool $(n=45)$ & $\mathbf{4 . 0 \pm 0 . 3 3}$ \\
PTB SUBJECTS ON ATT & Male $\quad(n=28)$ & $2.8 \pm 0.53$ \\
& Female $(n=17)$ & $2.9 \pm 0.29$ \\
CONTROL SUBJECTS & Pool $(n=45)$ & $\mathbf{2 . 8} \pm \mathbf{0 . 4 6}$ \\
& Male $(n=19)$ & $2.0 \pm 0.23$ \\
& Female $(n=25)$ & $1.9 \pm 0.32$ \\
& Pool $(n=44)$ & $\mathbf{2 . 0} \pm \mathbf{0 . 2 8}$ \\
\hline
\end{tabular}

$\mathrm{n}=$ number of subjects; MDA=Malondialdehyde; $\mathrm{ATT}=$ Anti tuberculosis treatment; $\mathrm{PTB}=\mathrm{Pulmonary}$ tuberculosis 
Table 2: Comparison between Serum levels of SOD in PTB subjects on drugs, those that have not started medications and control subjects

\begin{tabular}{lcl}
\hline Parameters & & Superoxide Dismutase $(\mathbf{I U} / \mathbf{m l})$ \\
\hline PTB SUBJECTS NOT ON ATT & $(\mathrm{n}=45)$ & $0.80 \pm 0.51$ \\
PTB SUBJECTS ON ATT & $(\mathrm{n}=45)$ & $1.45 \pm 0.48$ \\
CONTROL SUBJECTS & $(\mathrm{n}=44)$ & $2.05 \pm 0.85$ \\
\hline
\end{tabular}

$\mathrm{n}=$ number of subjects; $\mathrm{ATT}=$ Anti tuberculosis treatment; PTB=Pulmonary tuberculosis

\section{DISCUSSION}

Free radicals have been implicated in the development of lung fibrosis which may be a long-term development of pulmonary tuberculosis. They attack cell membrane, causing tissue damage and wasting disease in PTB patients. According to the results obtained from the present research work, patients with pulmonary tuberculosis that were not on Anti tuberculosis therapy $($ ATT $)$, had a statistically increased $(p<0.05)$ MDA mean values of $4.0 \pm 0.33$ compared to control subjects with MDA level of $2.0 \pm 0.23$. The increased MDA is signifying an ongoing oxidative stress whereby antioxidant substances might have been depleted at the expense of scavenging free radicals or stopping their propagation. Reddy et al., (2009) [11] and Hashmi et al., (2012) [13] reported a similar pattern of an increased MDA levels in PTB patients compared to control group. The result was also in agreement with findings by Samuel et al., (2013) [16] conducted in Ibadan, South-western Nigeria.

Accordingly, results obtained from this study, revealed statistically lower $(p<0.005)$ serum levels of SOD in PTB subjects without treatment than those on medications and control subjects. These findings were in line with the work of Mokogwu et al., (2016) [17]. Hashni et al (2012) [13] and Reddy et al., (2004) [11] whom also documented a significantly lower serum SOD in PTB subjects without anti PTB therapy. The abnormal decrease of SOD may be due increase utilization of SOD in clearing excess free radicals generated due to PTB.

\section{CONCLUSION}

The increased level of malondialdehyde shown by our study in pulmonary tuberculosis patients that were on drug and those that were not on drug as compared to the healthy individuals and decreased SOD further supported the relationship between tuberculosis infection and oxidative stress. It is an indication that Tuberculosis patients did not have enough antioxidants to fight up the free radicals generated which consequently provoked oxidation of membrane lipids leading to the production of high MDA as marker of oxidative stress. Therefore, treatment of tuberculosis through nutrition and anti-oxidant supplementation should be taken into consideration.

\section{Ethical Clearance}

Ethical clearance was given by Rasheed Shekoni Specialist Hospital Ethical Review Committee.

\section{Consent}

Informed consent was obtained from the patients or guardians (in case of the children) to participate in the study by signing or thumb-printing informed consent form. All samples collected were treated with utmost confidentiality.

\section{Acknowledgement}

We would like to thank the management of Rasheed Shekoni Specialist Hospital for their support. We also appreciate support given by staff of Medical Microbiology, Chemical Pathology as well as TB Clinic of Rasheed Shekoni for their support throughout the duration of this study.

\section{Conflict of Interest}

We declare that we have no conflict of interest.

\section{Financial Support}

None declared.

\section{REFERENCES}

1. Paton NI, Chua Y, Earnest A, Chee CBE. Randomized controlled trial of nutritional supplementation in patients with newly diagnosed tuberculosis and wasting. American Journal of Clinical Nutrition. 2004;(80):460-65.

2. WHO Report. Global Tuberculosis Control: Surveillance, Planning and Financing, 2008.

3. World Health Organization, Global Tuberculosis Report 2017.

4. Folaranmi OM, Adesiyan AA. Comparative study of plasma electrolytes ( $\mathrm{Na}, \mathrm{K}, \mathrm{Cl}$, and $\mathrm{HCO}_{3}$ ) and urea levels in HIV/AIDS and pulmonary tuberculosis infected subjects. Biokemistri. 2004;16(1):29-36.

5. Falzon D, Timimi H, Kurosinski P, Migliori GB, Van Gemert W, Denkinger $C$ et al. Digital health for the End TB Strategy: developing priority products and making them work. European Respiratory Journal. 2016;48(1):29-45.

6. Konstantinos A. Testing for tuberculosis. Australian Prescriber. 2010;33:12-18.

7. Gao F, Koenitzer JR, Tobolewski JM, Jiang D, Liang J, Noble PW et al. Extracellular superoxide dismutase inhibits inflammation by preventing oxidative fragmentation of hyaluronan. Journal of Biological Chemistry. 2008;283(10):6058-66.

8. Shing Dong-Ho, Sabrina S, Martinez M, Parsons DT, Jayaweera AC, Marriana KB. Relationship of oxidative Stress with HIV Disease progression in HIV/HCV co-infected and HIV monoinfected Adults in Maimi. Intl J Biosc Biochem Bioinforma. 2012; 2(3):217-223.

9. Lius Fernando D, Alexandro RL, Valdomaroe M. Oxidative stress and physical exercise in HCV Positive individuals. Rev. Bras Esporte. 2007:249-250.

10. Takujiro H, Junichi F. Dietary Interventions in Liver Disease, Foods, Nutrients and Dietary Supplements. Chapter 5 (2019):5970. Available online 8 February 2019.

11. Reddy YN, Murthy SV, Krishna DR, Prabhakar MC. Oxidative metabolic changes in pleural fluid of tuberculosis patients. Bangladesh J Pharmacol. 2009;4:69-72. 
12. Sudha K, Amareshwara M, Rakesh M. Study of protein oxidation and antioxidants status in pulmonary tuberculosis patients. International Journal of Pharma and Bio Sciences. 2011;2(3):1049 .

13. Hashmi MA, Ahsan B, Shah SI, Khan MI. Antioxidant capacity and lipid peroxidation product in pulmonary tuberculosis. Al Ameen $\mathrm{J}$ Med Sci. 2012;5(3):313-9.

14. Nadiger HA, Marcus SR, Chandrakala MV, Kulkarni DD. Malondialdehyde levels in different organs of rats subjected to acute alcohol toxicity. IJCB 1986;1:133-136.

15. Kakkar P, Das B, Viswanathan PN. A modified spectrophotometric assay of superoxide dismutase. Biochem. Biophys. 1984;21:130132.

16. Oyedeji SO, Adesina AA, Oke OT, Oguntuase NR, Esan A. Oxidative stress and lipid profile status in pulmonary tuberculosis patients in South Western Nigeria. Greener Journal of Medical Sciences. 2013;3(6):228-32.

17. Mokogwu AT, Airhomwanbor KO, Mokogwu EE, Onohwakpor EA. Lipid peroxidation and oxidative stress in pulmonary tuberculosis in Edo state, Nigeria. African Journal of Cellular Pathology. 2016;7:35-40. 